\title{
Étude expérimentale du branchement de fissure en présence de plusieurs types de frottement
}

\author{
Chineb Chaker ${ }^{1, a}$ et Mohsen Akrout ${ }^{2}$ \\ 1 Institut Préparatoire aux Études d'Ingénieur de Monastir, Rue Ibn El Jazzar, 5019 Monastir, Tunisie \\ 2 École Nationale des Ingénieurs de Sfax, Département de Mécanique, Route de Soukra Km 4, BP W 3038, Sfax, Tunisie
}

Reçu le 4 décembre 2002, accepté le 5 mars 2004

Résumé - On étudie un lot de plaquettes de PMMA avec branchements, obtenues sous compression uniaxiale pour un défaut oblique fermé par différents matériaux (Téflon, acier dur, bronze, laiton, acier doux, plomb, aluminium et cuivre) et faisant un angle d'inclinaison $\beta$ variable de 15 à $75^{\circ}$ par rapport à la direction de la charge imposée, en déterminant d'une part la distance $\delta$ entre le front de l'entaille et la racine du branchement mesurée parallèlement à l'axe de l'entaille, et d'autre part l'angle de branchement $\theta$ entre la direction de l'entaille et la tangente à la racine de la fissure branchée.

Mots clés : PMMA / compression / défaut oblique / différents matériaux / angle de branchement / fissure branchée

\begin{abstract}
Experimental study of the branch crack with presence of many type of friction. We study the PMMA plates with branch, formed under constant uniaxial loading for an oblique closed defect with different materials (Teflon, hard steel, bronze, brass, soft steel, lead, aluminium and copper) presenting different angle inclination $\beta$ ranging from 15 to $75^{\circ}$ with respect to the loading direction, in order to determine the distance $\delta$ from the branch crack root to the slot tip, and the branching angle $\theta$ between the slot direction and the tangent to the branch crack root.
\end{abstract}

Key words: PMMA / compression / oblique defect / different materials / branching angle / branch crack

\section{Nomenclature}

\begin{tabular}{|ll|}
\hline$\beta$ & Angle d'inclinaison du défaut \\
$\delta$ & $\begin{array}{l}\text { Distance entre le front de l'entaille et la racine } \\
\text { du branchement }\end{array}$ \\
$\theta$ & Angle de branchement \\
$\rho$ & Coefficient de frottement \\
$\mathrm{E}$ & Module d'Young \\
$\nu$ & Coefficient de Poisson \\
$\mathrm{G}$ & Taux de restitution d'énergie \\
$\mathrm{L}$ & Longueur de la fissure branchée \\
$\delta_{\mathrm{m}}$ & Valeur moyenne de la distance $\delta$ \\
$\theta_{\mathrm{m}}$ & Valeur moyenne de l'angle de branchement \\
$\theta_{\mathrm{c}}$ & Valeur critique de l'angle de branchement \\
\hline
\end{tabular}

a Auteur correspondant : Chiheb.Chaker@fsegs.rnu.tn

\section{Introduction}

Une série d'expériences de branchements en compression uniaxiale avait été réalisée sur des éprouvettes préentaillées de PMMA (polyméthacrylate de méthyle), matériau choisi pour son comportement fragile dans les conditions ordinaires, ses caractéristiques mécaniques bien déterminées, sa valeur en tant qu'analogue cassant de roches [1] et [2], et ductile lorsqu'il est soumis à de fortes contraintes différentielles [3]. Ces essais avaient porté principalement sur la localisation et l'orientation des branchements [4] et [5]. Ces essais avaient été réalisés sur des défauts ouverts (frottement nul), le branchement s'effectuant alors toujours perpendiculaire au plan tangent à la surface du défaut préexistant.

Le présent travail, s'inscrit dans la même démarche mais en prenant en compte l'influence du frottement entre les lèvres du défaut initial [6,7] (défaut fermé avec un seul type de matériau ayant un coefficient de frottement connu) [8] et [9], situation qui existe systématiquement dans les conditions confinées des milieux géologiques. 
On propose ici les résultats d'essais visant à étudier le problème de la localisation et de l'angle des branchements et de leur dépendance à l'inclinaison du défaut par rapport à la direction du chargement imposé et à l'intensité du frottement qui varie selon le matériau choisi (Téflon, acier dur, bronze, laiton, acier doux, plomb, aluminium ou cuivre).

\section{Dispositif expérimental}

Pour étudier la géométrie des fissures branchées, les essais ont été réalisés sur des plaquettes de dimensions $50 \times 32 \times 5 \mathrm{~mm}^{3}$ en PMMA (polyméthacrylate de méthyle) dont le module d'Young vaut $\mathrm{E}=2,9 \mathrm{GPa}$ et le coefficient de Poisson $\nu=0,4$. Dans un premier temps, nous avons déterminé le centre de la plaque, ensuite nous avons localisé la position de l'empreinte de l'entaille sur la plaque à l'aide d'une pointe à tracer et d'un rapporteur, puis nous avons percé un trou central de $0,4 \mathrm{~mm}$ de diamètre et enfin nous avons introduit la lame d'une scie d'horloger dans le trou central et nous avons effectué l'usinage de deux demi-entailles d'épaisseur e $=0,3 \mathrm{~mm}$ et de longueur $5 \mathrm{~mm}$ dans le prolongement l'une de l'autre après retournement de la lame scie. Pour effectuer cette opération, la lame de scie est guidée par deux lamelles en acier disposées de part et d'autre de l'éprouvette. Cette entaille est inclinée sous un angle $\beta$ variable allant de 15 à $75^{\circ}$ par rapport à la direction de la compression imposée. Cette entaille a été ensuite complètement colmatée à l'aide de très fines lamelles d'un matériau frictionnel afin de créer un défaut fermé avec un coefficient de frottement connu lors de la mise sous contrainte. Cette contrainte est imposée dans le sens de la plus grande dimension de l'éprouvette.

Pendant les essais, l'éprouvette était maintenue par deux raidisseurs latéraux en acier afin d'éviter le flambage, la pression était transmise par deux mâchoires crantées afin de réduire les effets de bord (Fig. 1). Les efforts étaient appliqués à l'aide d'un système à vis à servocommande. L'éprouvette est soumise à un échelon contrainte et le niveau du palier correspond à la contrainte d'initiation de la fissure branchée déterminée par des essais préalables. La rupture était obtenue sous chargement global constant.

\section{3 Évolution de la fissure branchée}

Quand $(\partial G / \partial L)>0$, l'équilibre est instable (sinon la fissure ne partirait pas), tandis que lorsque $(\partial G / \partial L)<0$, l'équilibre est stable : la fissure s'arrête [10] et [11], G étant le taux de restitution d'énergie et $L$ est la longueur de la fissure branchée. Pour l'évolution de la fissure branchée à partir d'un défaut initial sous contraintes uniaxiales croissantes, elle se dirige asymptotiquement dans la direction du chargement.

Un lot de plaquettes de PMMA avec branchements obtenues pour un défaut fermé par différents matériaux

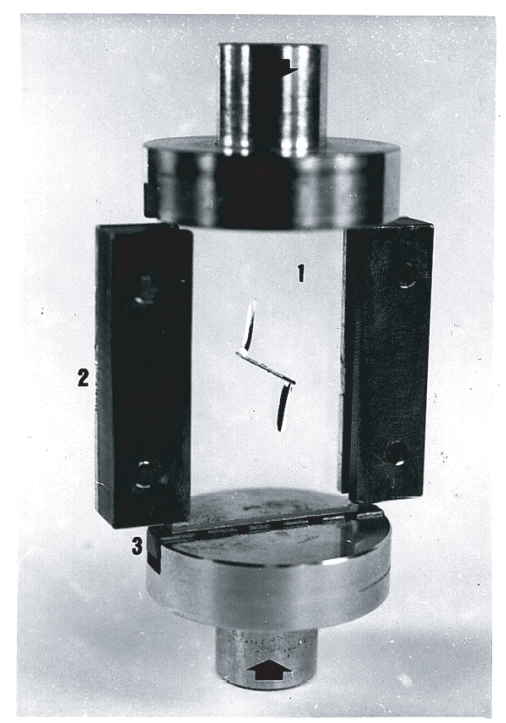

Fig. 1. Vue du dispositif expérimental : (1) éprouvette, (2) raidisseurs latéraux, (3) mâchoires crantées.

(Téflon, acier dur, bronze, laiton, acier doux, plomb, aluminium et cuivre) et faisant un angle d'inclinaison $\beta$ variable de 15 à $75^{\circ}$ par rapport à la direction de la charge imposée (Fig. 2a), a été examiné en déterminant d'une part la distance $\delta$ entre le front de l'entaille et la racine du branchement mesurée parallèlement à l'axe de l'entaille, et d'autre part l'angle de branchement $\theta$ entre la direction de l'entaille et la tangente à la racine de la fissure branchée (Fig. 2b).

\section{Résultats des essais et discussion}

En appliquant le chargement uniaxial, on observe l'apparition d'une fissure branchée au voisinage d'une extrémité de l'entaille. Cette fissure s'initie à partir d'une des nombreuses micro-fissures induites par le sciage. En général, après deux à trois secondes, une deuxième fissure branchée se développe vers l'autre extrémité du défaut. Ensuite, les deux fissures branchées, qui s'initient symétriquement par rapport au centre du défaut, se propagent lentement suivant des trajectoires qui s'incurvent plus ou moins selon l'inclinaison du défaut initial. Les fissures branchées restent parfaitement symétriques par rapport au centre de l'entaille et rejoignent la direction de la contrainte appliquée avant de s'arrêter, tandis que se déploie dans le prolongement du défaut imposé une zone plastique qui correspond à une zone de cisaillement plus ou moins ductile se développant par suite d'une forte surcontrainte différentielle bien repérable en photoélasticité.

\section{1 Étude de la fissure pour un défaut fermé par du Téflon}

Un lot de plaquettes de PMMA, ayant un angle d'inclinaison $\beta$ allant de 15 à $75^{\circ}$ par rapport à la direction 

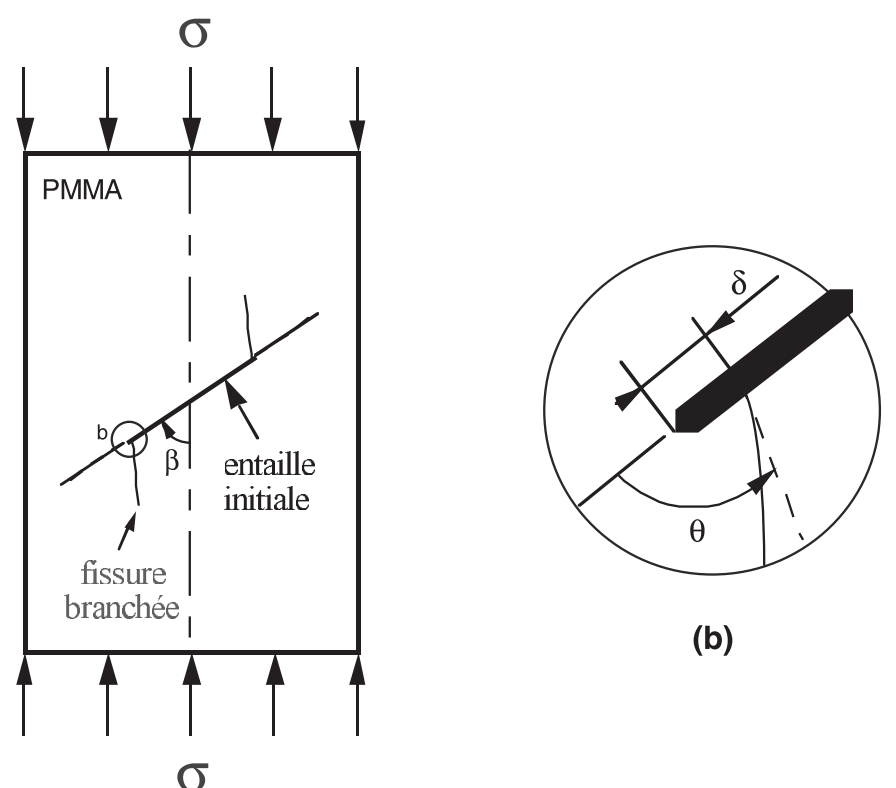

(a)

Fig. 2. (a) Plaquette de PMMA avec un défaut oblique traversant fermé faisant un angle $\beta$ avec l'axe de chargement uniaxial. (b) Schéma définissant l'angle de branchement $\theta$ et la distance $\delta$ de la racine de la fissure branchée à l'extrémité du défaut.

de la charge imposée, a été étudié en colmatant l'entaille par du Téflon afin de créer un défaut fermé (Téflon contre lui-même) avec un coefficient de frottement bas $\rho=0,11$ lors de la mise sous contrainte.

Afin d'étudier la position du branchement de la fissure, on a mesuré la distance $\delta$ du front de l'entaille à la racine $\mathrm{du}$ branchement. Ces mesures nous ont permis de constater que pour les défauts d'inclinaisons $\beta$ croissantes de 15 à $75^{\circ}$, les fissures sont initiées à une distance $\delta$ très proche du front de l'entaille et qui augmente avec l'inclinaison du défaut $\beta$ (Fig. 3). À titre indicatif, la valeur moyenne de la distance $\delta$ pour $\beta=30^{\circ}$ étant égale à $\delta_{\mathrm{m}}=0,175 \mathrm{~mm}$ et pour $\beta=75^{\circ}$, la valeur moyenne de la distance $\delta$ est $\delta_{\mathrm{m}}=0,330 \mathrm{~mm}$ (Fig. 3). Ces résultats montrent que la distance $\delta$ augmente faiblement avec l'inclinaison $\beta$ du défaut, comme dans le cas d'un défaut ouvert (frottement nul) ce qui n'est pas étonnant du fait de la valeur faible du coefficient de frottement.

Concernant la mesure de l'angle de branchement $\theta$, défini communément par l'angle que fait la direction de l'entaille initiale avec la tangente à la racine de la fissure branchée, on a pu constater que pour des inclinaisons $\beta$ allant de 15 à $45^{\circ}$, l'angle de branchement $\theta$ croît avec les inclinaisons $\beta$ (la valeur moyenne de l'angle de branchement $\theta_{\mathrm{m}}=81^{\circ}, 05$ pour $\beta=45^{\circ}$ ). Pour la valeur moyenne de l'angle de branchement $\theta$, laquelle augmente avec l'inclinaison $\beta$, les dispersions des mesures des deux paramètres $\delta$ et $\beta$ étant de même amplitude dans les deux cas, défaut ouvert et défaut fermé avec frottement, ce qui change du fait de l'existence d'un cisaillement au niveau des lèvres fermées du défaut initial, conduisant à
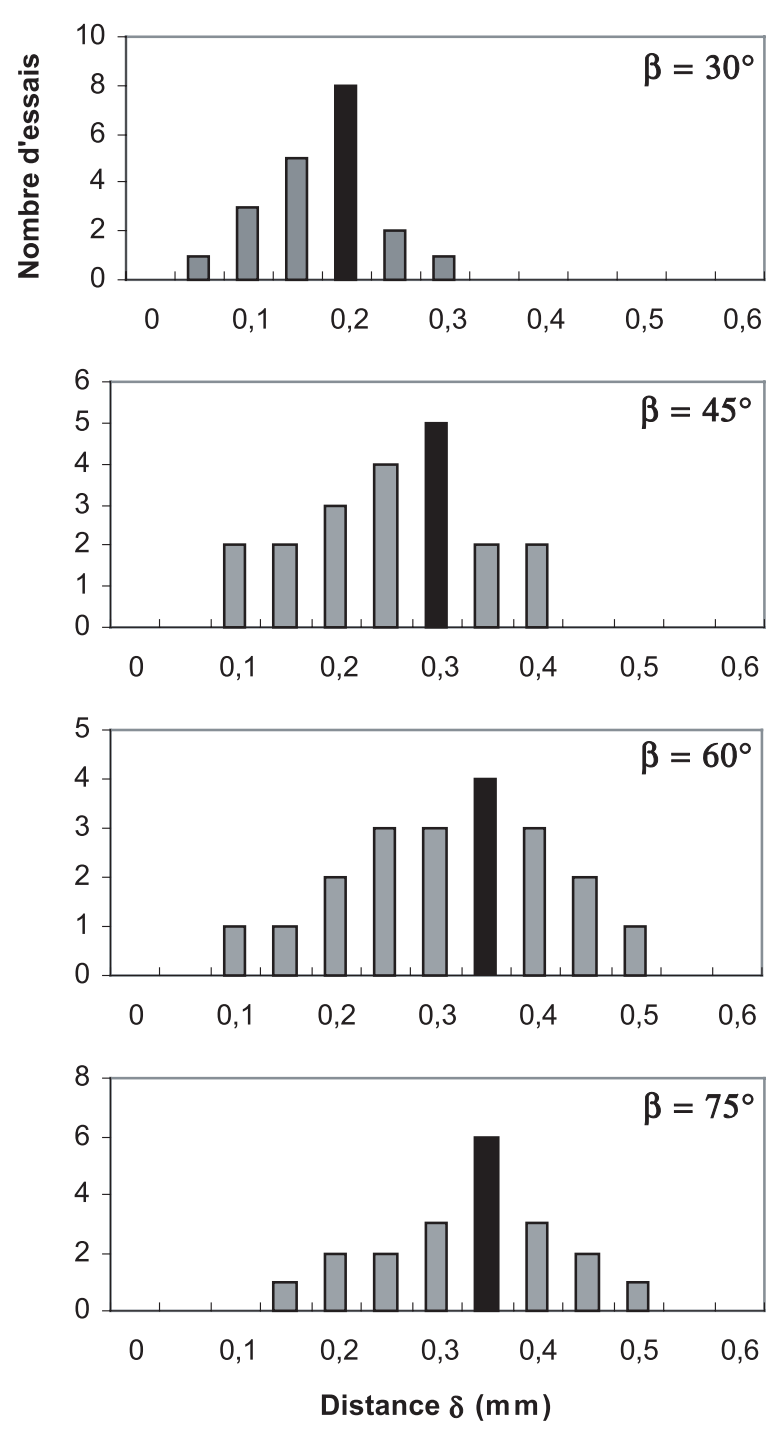

Fig. 3. Histogrammes représentant la distance $\delta$ pour les inclinaisons $\beta$ allant de 30 à $75^{\circ}$ en présence de Téflon $(\rho=0,11)$.

un coefficient de frottement évalué à $\rho=0,11$ (pour le Téflon) à l'aide d'une mesure parallèle, c'est la valeur limite $\theta_{\mathrm{c}}$ atteinte par l'angle de branchement $\theta$. Cet angle $\theta$ qui tendait rapidement vers $90^{\circ}$ en absence de frottement (angle que fait normalement une isostatique de contrainte principale débouchant sur un bord libre) tend ici asymptotiquement vers la valeur critique $\theta_{c}$ laquelle dépend de la nature du matériau. Cet angle critique $\theta_{c}$ étant la valeur maximale de l'angle de branchement $\theta$. Pour les inclinaisons $\beta=60$ et $75^{\circ}$, il a été trouvé que l'angle de branchement $\theta$ augmente faiblement et tend vers la valeur critique $\theta_{\mathrm{c}}=84 \pm 1^{\circ}$ par rapport à l'axe de l'entaille (Fig. 4), alors que pour un défaut ouvert (sans frottement : $\rho=0) \theta_{\mathrm{c}}=90 \pm 1^{\circ}$. En d'autres termes, la valeur moyenne de l'angle de branchement $\theta$ croît avec l'angle d'inclinaison $\beta$ et tend asymptotiquement vers la valeur critique $\theta_{c}$ mise en évidence. Ainsi, on voit clairement l'influence du frottement sur l'initiation de la fissure branchée déterminée par l'angle de branchement $\theta$. 


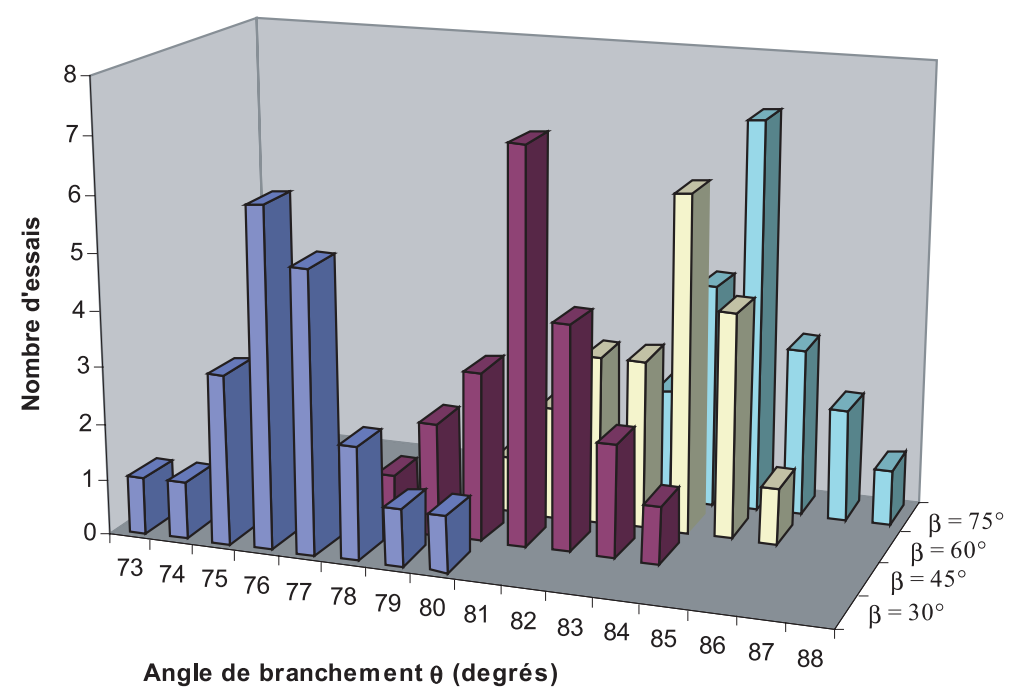

Fig. 4. Histogrammes représentant l'angle de branchement $\theta$ pour les inclinaisons $\beta$ variables de 30 à $75^{\circ}$ en présence de Téflon $(\rho=0,11)$.

\section{2 Étude de la fissure pour un défaut fermé par de l'acier dur}

On a utilisé également de l'acier dur qui induit un coefficient de frottement $\rho=0,13$ lors de la mise sous contrainte. Cette étude a été réalisée dans les mêmes conditions qu'avec le Téflon.

La mesure de la distance $\delta$ du front de l'entaille à la racine du branchement, permet de constater que pour les défauts d'inclinaisons $\beta=15$ et $30^{\circ}$, l'initiation tend à se produire au voisinage immédiat du front de l'entaille $\delta \leq 0,3 \mathrm{~mm}$ (pour $\beta=30^{\circ}$, la valeur moyenne de la distance est $\left.\delta_{\mathrm{m}}=0,200 \mathrm{~mm}\right)$. En revanche, pour les inclinaisons $\beta=45,60$ et $75^{\circ}$, les fissures sont initiées à une distance moyenne $\delta$ du front de l'entaille au moins égale à l'épaisseur de cette entaille, c'est-à-dire $\delta \geq 0,3 \mathrm{~mm}$ (Fig. 5). À titre indicatif, la valeur moyenne de la distance pour $\beta=60$ et $75^{\circ}$ est respectivement $\delta_{\mathrm{m}}=0,315$ et $0,332 \mathrm{~mm}$. On a également observé, comme dans le cas d'un défaut ouvert, que la distance $\delta$ augmente avec l'inclinaison $\beta$ du défaut.

Pour la mesure de l'angle de branchement $\theta$ entre la direction de l'entaille initiale et la tangente à la racine de la fissure branchée, on a pu constater que pour des inclinaisons $\beta$ allant de 15 à $45^{\circ}$, l'angle de branchement $\theta$ croît avec l'inclinaison $\beta$ à partir de la valeur moyenne $\theta_{\mathrm{m}}=54^{\circ}, 35$ pour $\beta=15^{\circ}$ jusqu'à $\theta_{\mathrm{m}}=80^{\circ}, 65$ pour $\beta=45^{\circ}$. Pour les inclinaisons $\beta=60$ et $75^{\circ}$, on a trouvé que l'angle de branchement $\theta$ augmente faiblement et tend vers la valeur critique $\theta_{\mathrm{c}}=84 \pm 1^{\circ}$ par rapport à l'axe de l'entaille (Fig. 6).

\section{3 Étude de la fissure pour un défaut fermé par du bronze}

Le frottement bronze-bronze fournit le coefficient $\rho=$ 0,16 . En mesurant la distance $\delta \mathrm{du}$ front de l'entaille à
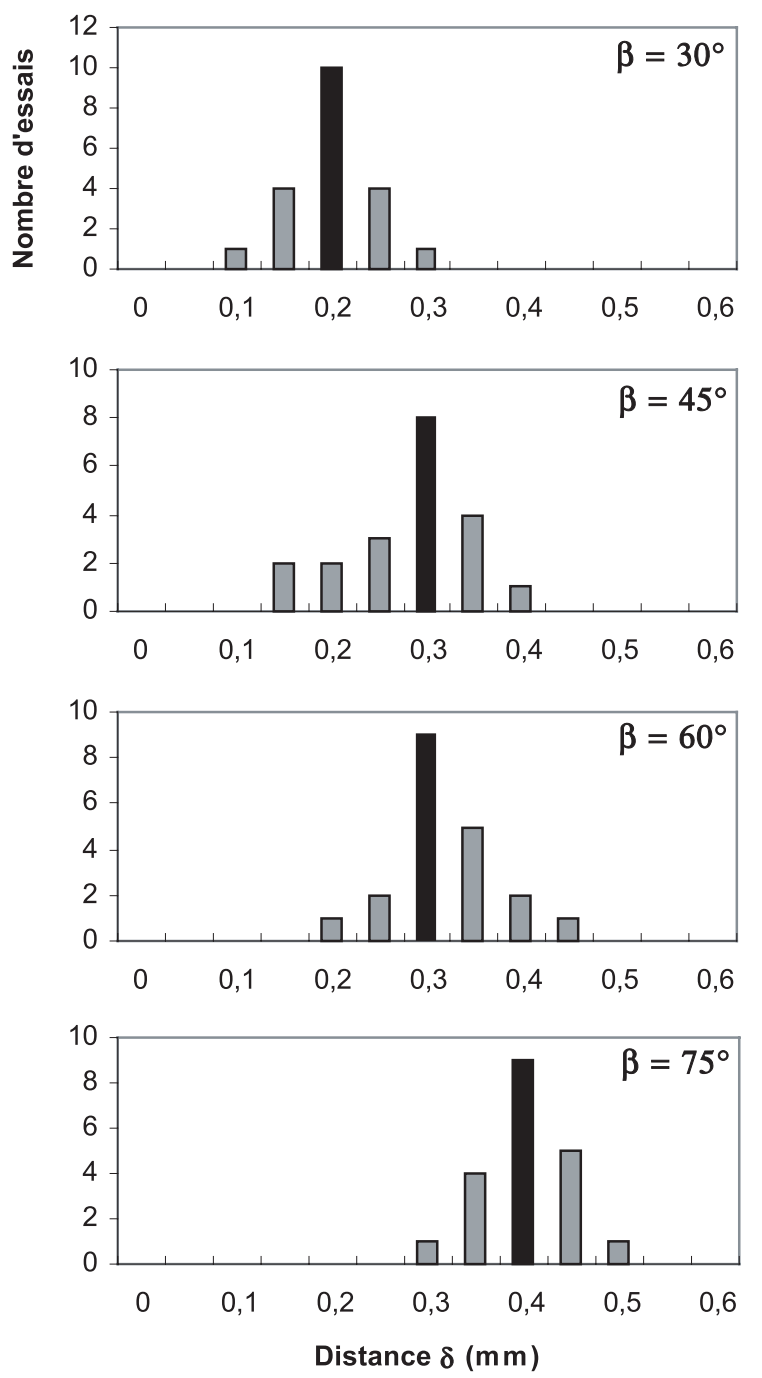

Fig. 5. Histogrammes représentant la distance $\delta$ pour les inclinaisons $\beta$ allant de 30 à $75^{\circ}$ en présence de l'acier dur $(\rho=0,13)$. 


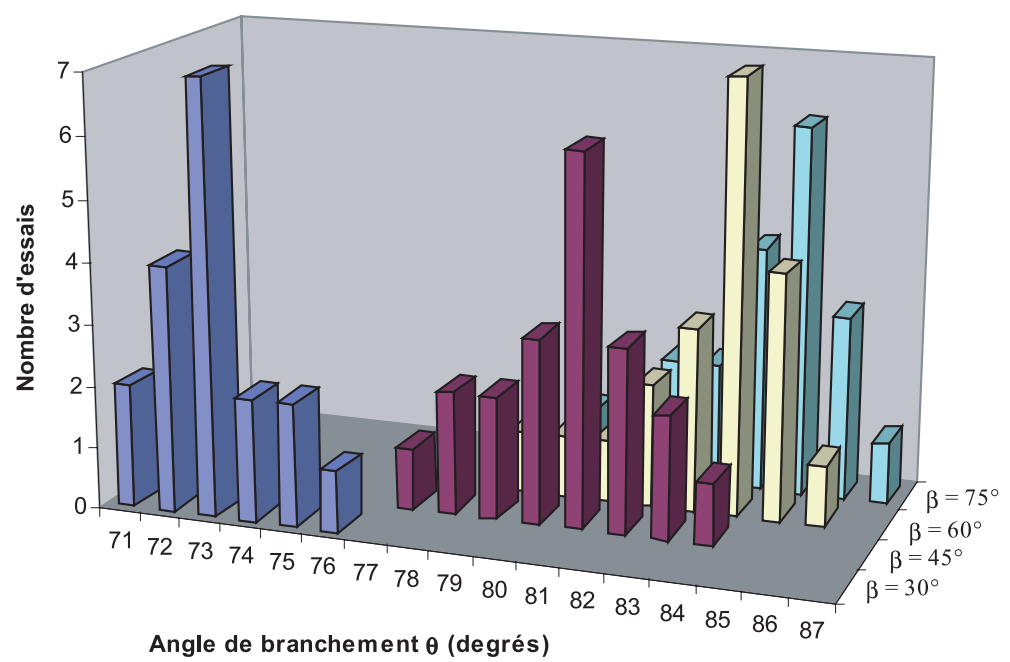

Fig. 6. Histogrammes représentant l'angle de branchement $\theta$ pour les inclinaisons $\beta$ variables de 30 à $75^{\circ}$ en présence de l'acier $\operatorname{dur}(\rho=0,13)$.

la racine du branchement, on a remarqué que pour les défauts d'inclinaisons $\beta=15,30$ et $45^{\circ}$, l'initiation tend à se produire au voisinage immédiat du front de l'entaille $\delta \leq 0,3 \mathrm{~mm}$ (pour $\beta=30^{\circ}$, la valeur de la distance moyenne est $\left.\delta_{\mathrm{m}}=0,246 \mathrm{~mm}\right)$. Mais pour les inclinaisons $\beta=60$ à $75^{\circ}$, les fissures sont initiées à une distance moyenne $\delta \mathrm{du}$ front de l'entaille supérieure ou égale à l'épaisseur de cette entaille $\delta \geq 0,3 \mathrm{~mm}$ (Fig. 7). Donc, on a la distance $\delta$ augmente avec l'inclinaison $\beta$ du défaut.

Pour la mesure de l'angle de branchement $\theta$, défini communément par l'angle que fait la direction de l'entaille initiale avec la tangente à la racine de la fissure branchée, il a été trouvé que pour des inclinaisons $\beta$ du défaut allant de 15 à $75^{\circ}$, l'angle de branchement $\theta$ croît avec l'inclinaison $\beta$ à partir de la valeur moyenne $\theta_{\mathrm{m}}=72^{\circ}, 6$ pour $\beta=30^{\circ}$ jusqu'à $\theta_{\mathrm{m}}=83^{\circ}, 9$ pour $\beta=75^{\circ}$ (Fig. 8 ). De plus, on a remarqué que l'angle de branchement $\theta$ augmente faiblement pour $\beta=60$ et $75^{\circ}$ et tend vers la valeur critique $\theta_{\mathrm{c}}=83 \pm 1^{\circ}$ par rapport à la direction de l'entaille (Fig. 8).

\section{4 Étude de la fissure pour un défaut fermé par du laiton}

Le frottement laiton-laiton donne le même coefficient que le frottement bronze-bronze $\rho=0,16$. En examinant dans un premier temps la distance $\delta$ du front de l'entaille à la racine du branchement, on a constaté que pour des angles d'inclinaison faibles, les fissures sont initiées à une distance $\delta$ voisine du front de l'entaille (la valeur moyenne de la distance est $\delta_{\mathrm{m}}=0,1 \mathrm{~mm}$ c'est-à-dire le tiers de l'épaisseur de l'entaille pour $\beta=15^{\circ}$ et $\delta_{\mathrm{m}}=0,250 \mathrm{~mm}$ pour $\beta=30^{\circ}$ ). Tandis que pour les inclinaisons $\beta \mathrm{du}$ défaut allant de 45 à $75^{\circ}$, les fissures sont initiées le plus fréquemment à une distance $\delta$ du front d'entaille au moins égale à l'épaisseur de l'entaille $\delta \geq 0,3 \mathrm{~mm}$ (Fig. 9). Donc la distance $\delta$ augmente toujours avec l'inclinaison $\beta$ du défaut.
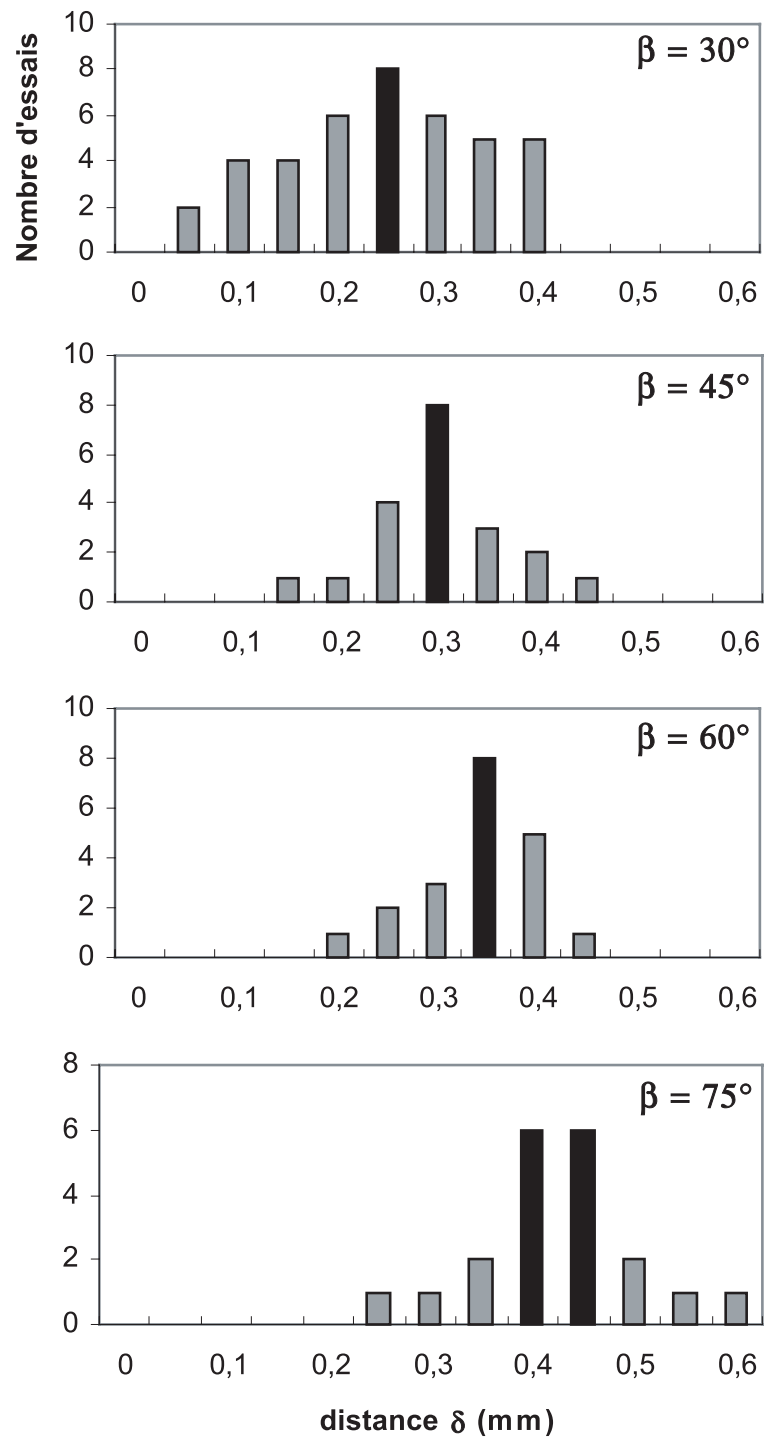

Fig. 7. Histogrammes représentant la distance $\delta$ pour les inclinaisons $\beta$ allant de 30 à $75^{\circ}$ en présence de bronze $(\rho=0,16)$. 


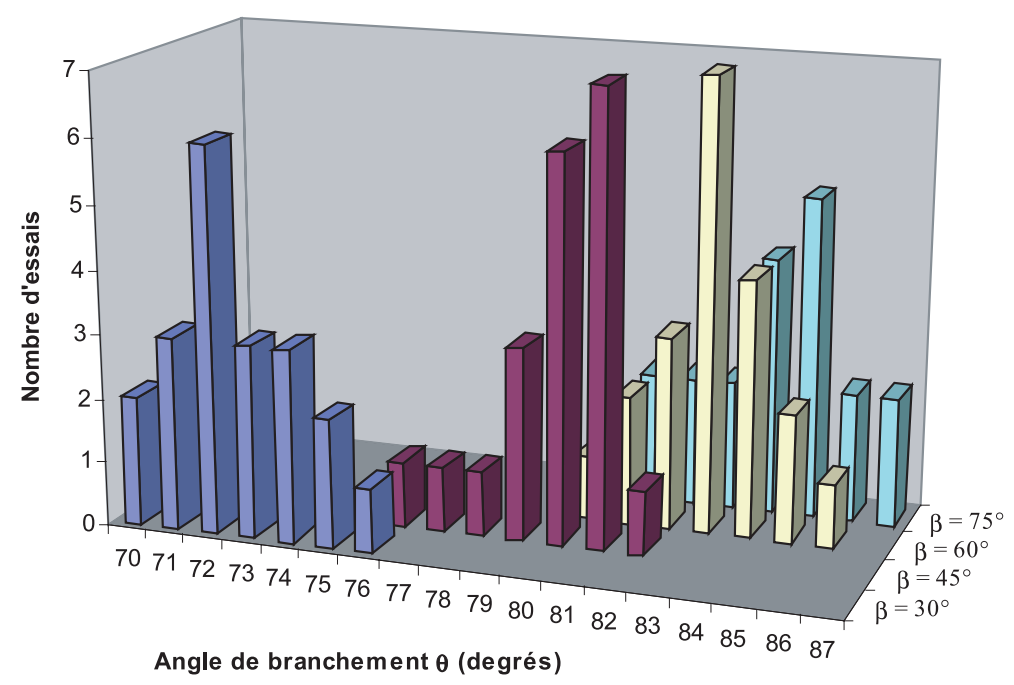

Fig. 8. Histogrammes représentant l'angle de branchement $\theta$ pour les inclinaisons $\beta$ variables de 30 à $75^{\circ}$ en présence de bronze $(\rho=0,16)$.
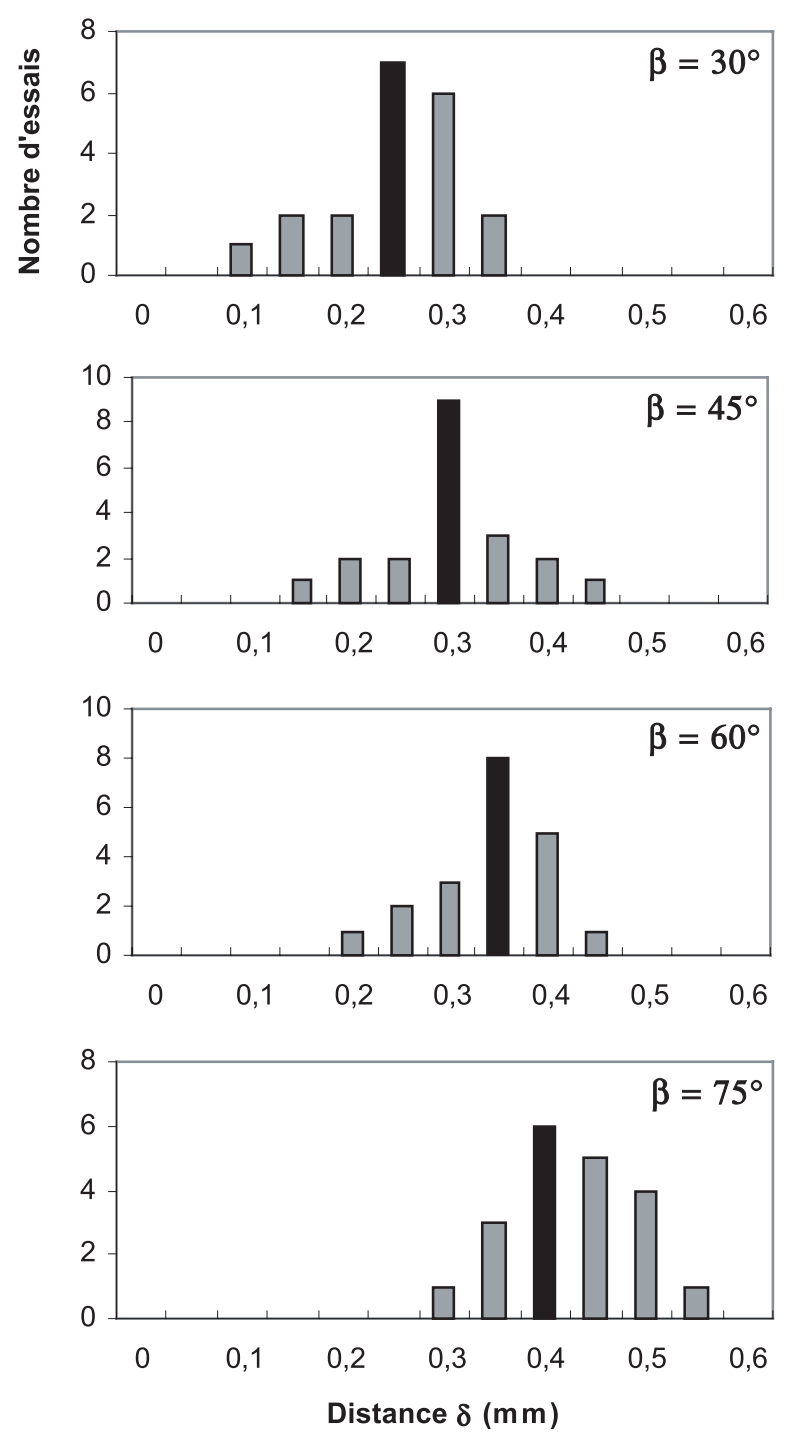

Fig. 9. Histogrammes représentant la distance $\delta$ pour les inclinaisons $\beta$ allant de 30 à $75^{\circ}$ en présence de laiton $(\rho=0,16)$.
Après en mesurant l'angle de branchement $\theta$ entre la direction de l'entaille initiale et la tangente à la racine de la fissure branchée, on a pu constater que pour des inclinaisons $\beta$ du défaut allant de 15 à $45^{\circ}$, l'angle de branchement $\theta$ croît avec l'inclinaison $\beta$ à partir de la valeur moyenne $\theta_{\mathrm{m}}=52^{\circ}, 35$ pour $\beta=15^{\circ}$ jusqu'à $\theta_{\mathrm{m}}=$ $79^{\circ}, 25$ pour $\beta=45^{\circ}$. Mais pour les inclinaisons $\beta=60$ et $75^{\circ}$, l'angle de branchement $\theta$ augmente faiblement et tend vers la valeur critique $\theta_{\mathrm{c}}=83 \pm 1^{\circ}$ par rapport à l'axe de l'entaille (Fig. 10), alors que pour un défaut ouvert (frottement nul) la fissure branchée s'initie avec un angle de branchement qui tend rapidement vers $\theta_{\mathrm{c}}=90 \pm 1^{\circ}$.

\section{5 Étude de la fissure pour un défaut fermé par de l'acier doux}

Le frottement de l'acier doux contre lui-même fournit le coefficient $\rho=0,17$. Dans l'étude expérimentale, on a mesuré la distance $\delta$ du front de l'entaille à la racine $\mathrm{du}$ branchement et on a constaté que, pour $\beta=15^{\circ}$ la fissure est initiée au voisinage immédiat du bout de l'entaille, sa valeur moyenne étant $\delta_{\mathrm{m}}=0,115 \mathrm{~mm}$, mais pour les inclinaisons $\beta$ allant de 30 à $75^{\circ}$, les fissures sont initiées à une distance du front de l'entaille supérieure ou égale à l'épaisseur de l'entaille (Fig. 11). À titre indicatif, la valeur moyenne de la distance $\delta$ pour $\beta=45^{\circ}$ étant égale à $0,315 \mathrm{~mm}$. Cette distance $\delta$ augmente faiblement avec l'inclinaison $\beta$ du défaut et ne dépasse pas la valeur 0,45 mm (Fig. 11).

Concernant la mesure de l'angle de branchement $\theta$, défini communément par l'angle que fait la direction de l'entaille initiale avec la tangente à la racine de la fissure branchée, on a constaté que cet angle de branchement $\theta$ croît avec les inclinaisons $\beta$ du défaut allant de 15 à $45^{\circ}$ (la valeur moyenne l'angle de branchement $\theta$ pour $\beta=45^{\circ}$ étant égal à $\left.79^{\circ}, 15\right)$. Par contre pour les inclinaisons du défaut $\beta=60$ et $75^{\circ}$, on a trouvé que l'angle de branchement $\theta$ augmente faiblement et tend 


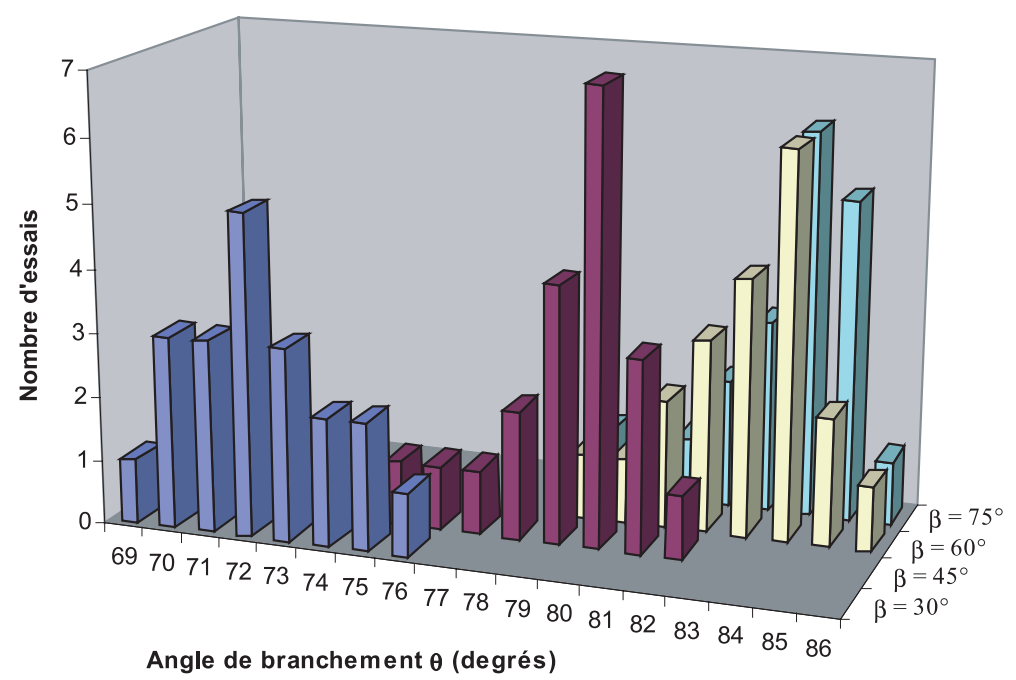

Fig. 10. Histogrammes représentant l'angle de branchement $\theta$ pour les inclinaisons $\beta$ variables de 30 à $75^{\circ}$ en présence de laiton $(\rho=0,16)$.
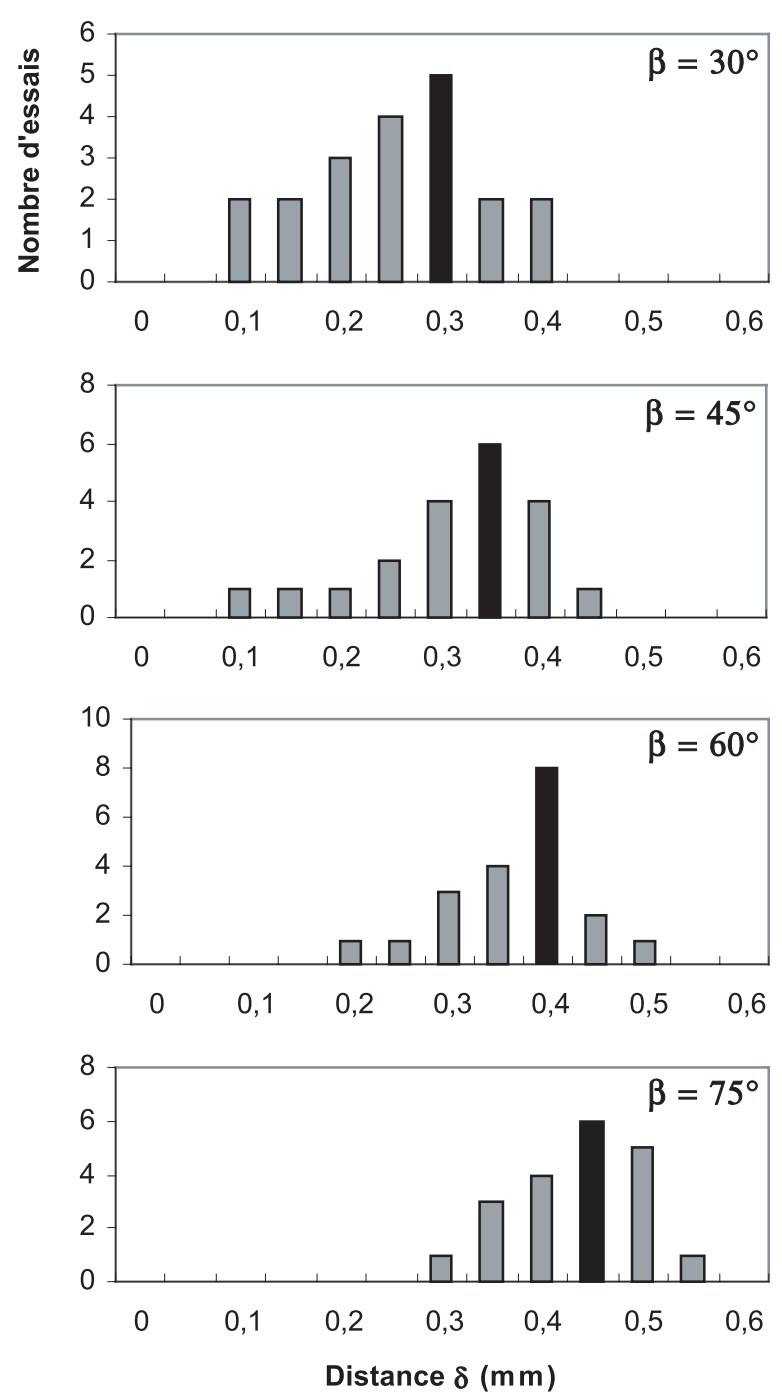

Fig. 11. Histogrammes représentant la distance $\delta$ pour les inclinaisons $\beta$ allant de 30 à $75^{\circ}$ en présence de l'acier doux $(\rho=0,17)$. vers la valeur critique $\theta_{\mathrm{c}}=82 \pm 1^{\circ}$ par rapport à l'axe de l'entaille (Fig. 12). Aussi, on a remarqué que l'angle de branchement $\theta$ est en train de diminuer en augmentant le coefficient de frottement $\rho$.

\section{6 Étude de la fissure pour un défaut fermé par du plomb}

Notre étude a porté aussi sur un défaut fermé à l'aide des plaquettes fines de plomb avec un coefficient de frottement $(\rho=0,17)$ plomb contre plomb. Il s'agit de la même valeur que pour le frottement de l'acier doux.

Dans cette étude, on a mesuré la distance $\delta$ du front de l'entaille à la racine du branchement et on a remarqué que pour les défauts d'inclinaisons $\beta=15,30^{\circ}$, les fissures branchées sont initiées à une distance $\delta$ proche du front de l'entaille et ne dépasse pas $0,3 \mathrm{~mm}$. Tandis que, pour les inclinaisons $\beta=45,60$ et $75^{\circ}$, l'initiation des fissures s'éloigne légèrement du front de l'entaille et débute à une distance $\delta$ supérieure ou égale à l'épaisseur de l'entaille c'est-à-dire $\delta \geq 0,3 \mathrm{~mm}$ (Fig. 13). À titre indicatif, la valeur moyenne de la distance $\delta$ respectivement pour $\beta=$ $45^{\circ}$ et $75^{\circ}$ étant égale à $\delta_{\mathrm{m}}=0,320 \mathrm{~mm}$ et $0,450 \mathrm{~mm}$. On a remarqué aussi que la distance $\delta$ croît avec l'inclinaison $\beta$ du défaut et avec le coefficient de frottement $\rho$.

Pour ce qui concerne la mesure de l'angle de branchement $\theta$, entre la direction de l'entaille initiale et la tangente à la racine de la fissure branchée, on a constaté que pour des inclinaisons $\beta=15$ et $30^{\circ}$, l'angle de branchement $\theta$ croît avec l'inclinaison $\beta$ (la valeur moyenne de l'angle de branchement est $\theta_{\mathrm{m}}=50^{\circ}, 55$ pour $\beta=15^{\circ}$ et $\theta_{\mathrm{m}}=70^{\circ}, 1$ pour $\beta=30^{\circ}$ ). Mais pour les inclinaisons $\beta$ du défaut allant de 45 à $75^{\circ}$, on a pu trouver que l'angle de branchement $\theta$ augmente faiblement et tend vers la valeur critique $\theta_{\mathrm{c}}=82 \pm 1^{\circ}$ par rapport à l'axe de l'entaille (Fig. 14). 


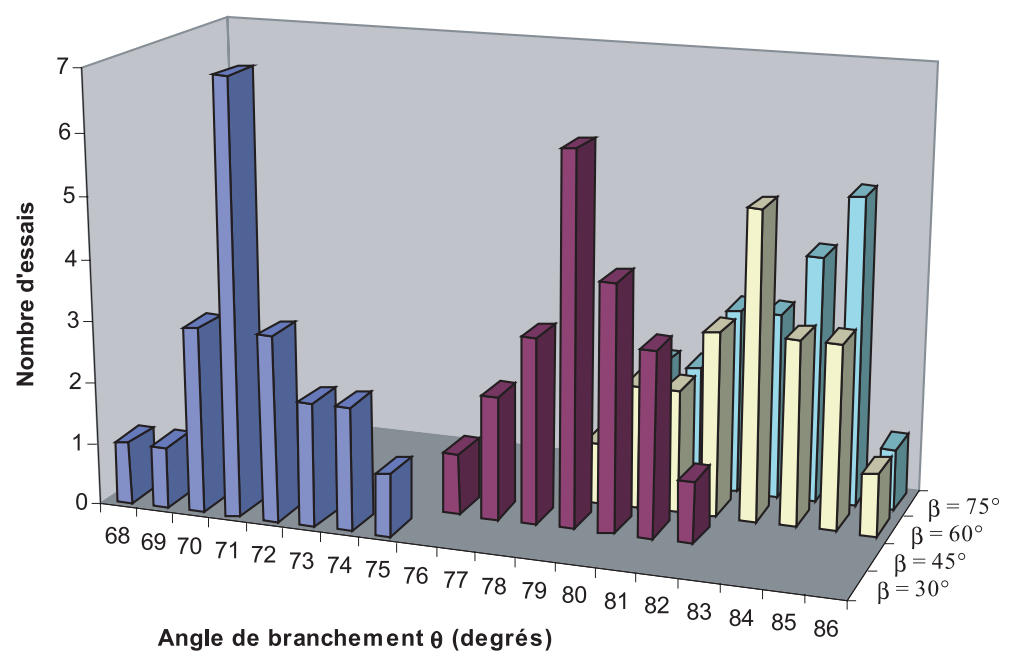

Fig. 12. Histogrammes représentant l'angle de branchement $\theta$ pour les inclinaisons $\beta$ variables de 30 à $75^{\circ}$ en présence de l'acier doux $(\rho=0,17)$.
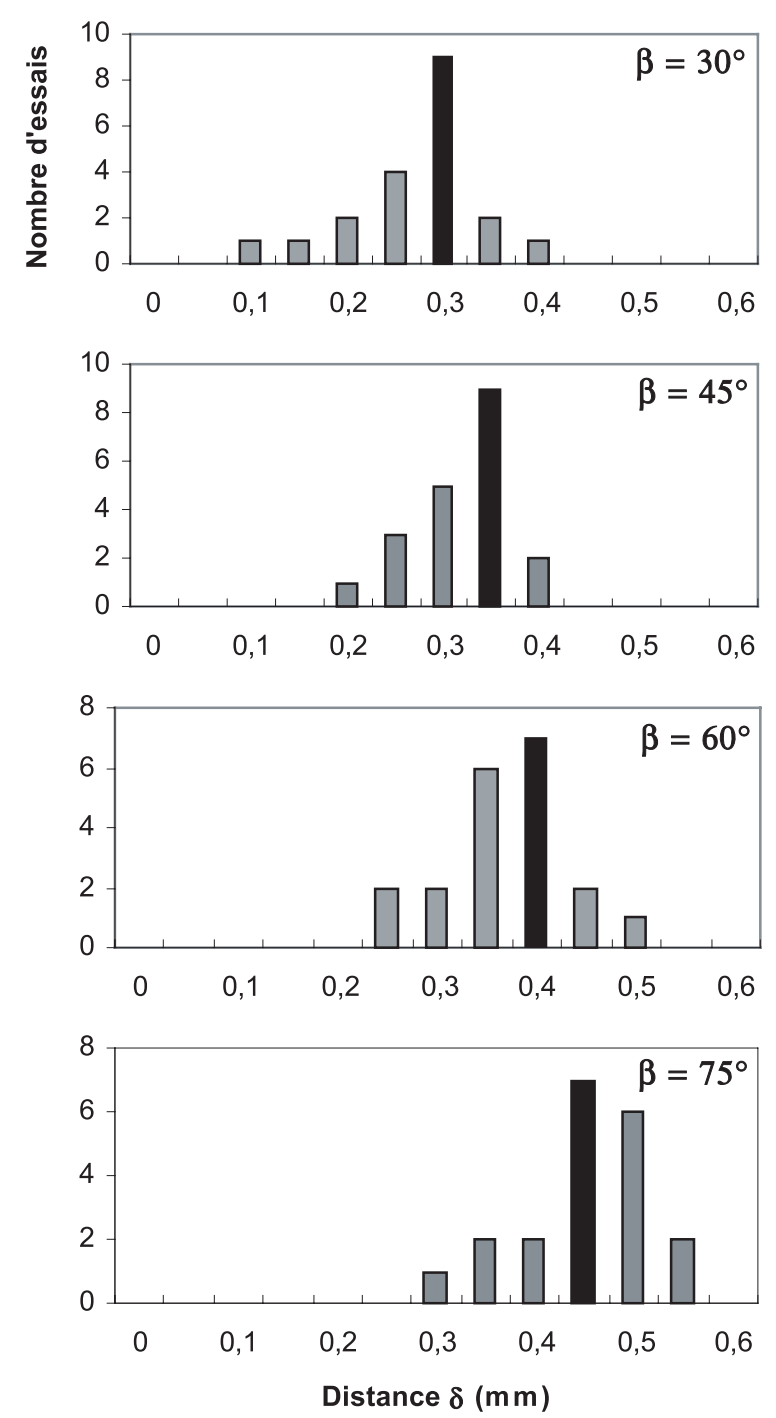

Fig. 13. Histogrammes représentant la distance $\delta$ pour les inclinaisons $\beta$ allant de 30 à $75^{\circ}$ en présence de plomb $(\rho=$ $0,17)$.

\section{7 Étude de la fissure pour un défaut fermé par de l'aluminium}

On a examiné également un lot de plaquettes de PMMA avec branchements obtenus en créant un défaut fermé (frottement aluminium contre lui même) avec un coefficient de frottement $\rho=0,18$ en colmatant l'entaille par des plaquettes très minces d'aluminium. Ce défaut a été étudié pour des angles d'inclinaison $\beta$ variables de 15 à $75^{\circ}$ par rapport à la direction de la compression.

Cette étude nous a permis d'abord de déterminer la distance $\delta \mathrm{du}$ front de l'entaille à la racine du branchement, dans ce cas on a pu constaté que pour les défauts d'inclinaisons $\beta$ allant de 15 à $30^{\circ}$, l'initiation tend à se produire au voisinage immédiat du front de l'entaille, cette distance $\delta$ étant inférieure ou égale à l'épaisseur de l'entaille c'est-à-dire $\delta \leq 0,3 \mathrm{~mm}$ (pour $\beta=30^{\circ}$ la valeur moyenne de la distance étant égale à $0,280 \mathrm{~mm}$ ). Mais pour les inclinaisons $\beta=45,60$ et $75^{\circ}$, les fissures sont initiées à une distance $\delta \mathrm{du}$ front de l'entaille supérieure ou égale à l'épaisseur de cette entaille $\delta \geq 0,3 \mathrm{~mm}$ (Fig. 15). À titre indicatif, la valeur moyenne de la distance $\delta_{\mathrm{m}}=0,350 \mathrm{~mm}$ pour $\beta=45^{\circ}$ et $\delta_{\mathrm{m}}=0,460 \mathrm{~mm}$ pour $\beta=75^{\circ}$ (Fig. 15).

Ensuite, on a déterminé l'angle de branchement $\theta$, défini communément par l'angle que fait la direction de l'entaille initiale avec la tangente à la racine de la fissure branchée, qui d'une part pour les inclinaisons $\beta$ allant de 15 à $45^{\circ}$, croît avec l'inclinaison $\beta$ à partir de la valeur moyenne $\theta_{\mathrm{m}}=43^{\circ}, 85$ pour $\beta=15^{\circ}$ jusqu'à $\theta_{\mathrm{m}}=77^{\circ}$ pour $\beta=45^{\circ}$, et d'autre part pour les inclinaisons $\beta=60$ et $75^{\circ}$, on a pu constater que l'angle de branchement $\theta$ augmente faiblement et tend vers la valeur critique $\theta_{\mathrm{c}}=84 \pm 1^{\circ}$ par rapport à l'axe de l'entaille (Fig. 16). 


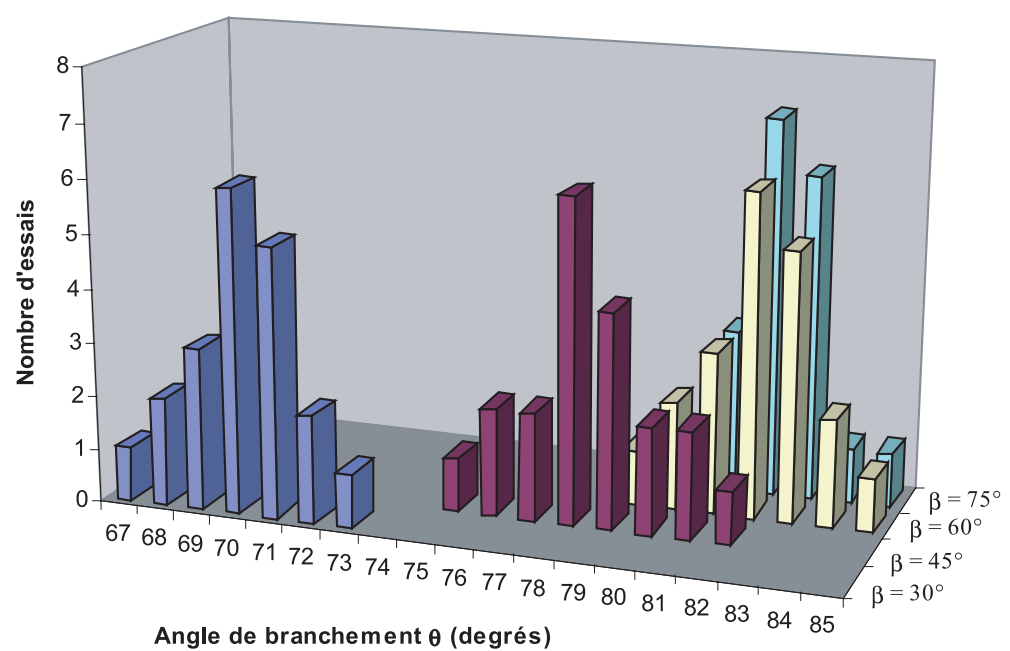

Fig. 14. Histogrammes représentant l'angle de branchement $\theta$ pour les inclinaisons $\beta$ variables de 30 à $75^{\circ}$ en présence de plomb $(\rho=0,17)$.
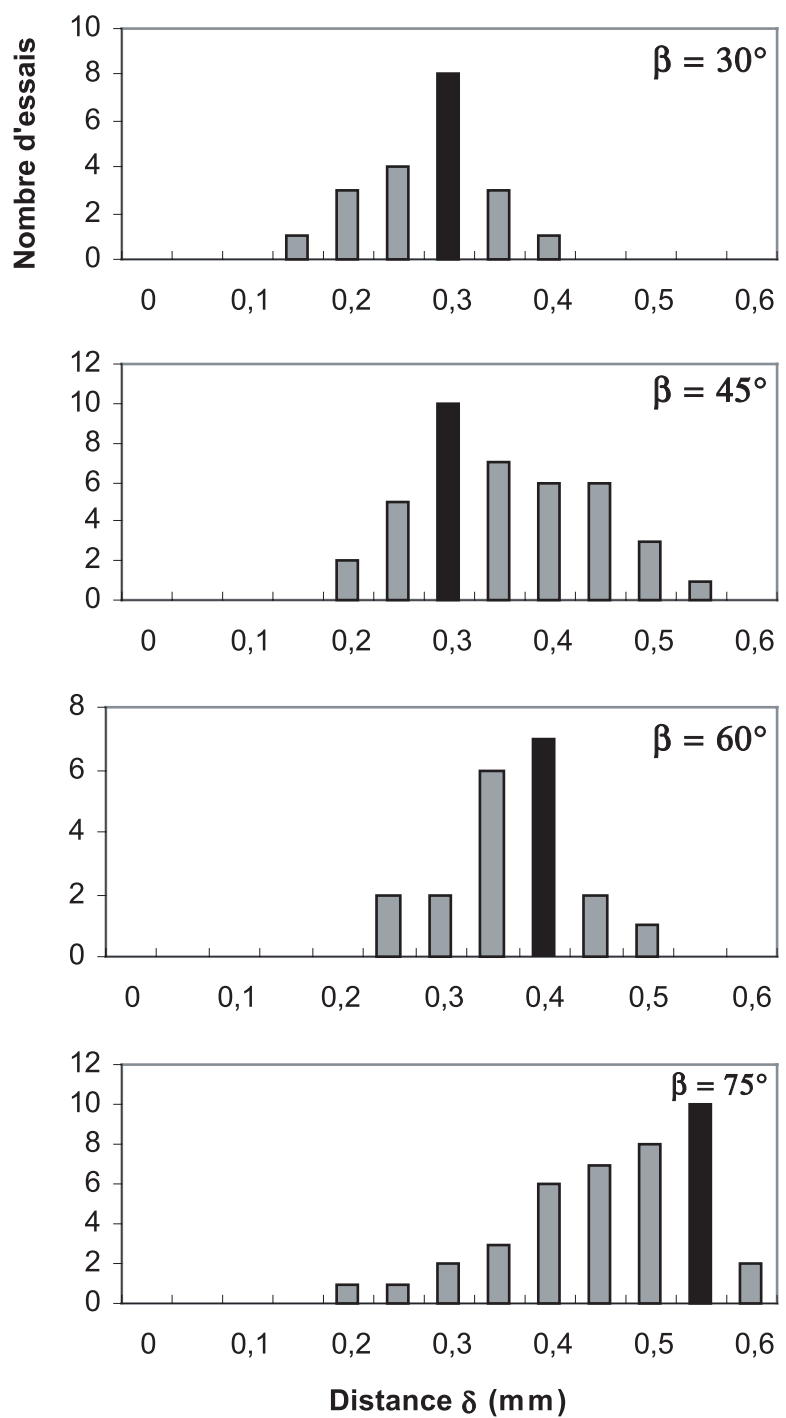

Fig. 15. Histogrammes représentant la distance $\delta$ pour les inclinaisons $\beta$ allant de 30 à $75^{\circ}$ en présence d'aluminium ( $\rho=$ $0,18)$.

\section{8 Étude de la fissure pour un défaut fermé par du cuivre}

Enfin, notre défaut est fermé par des plaquettes très fines de cuivre afin de créer un frottement cuivre contre cuivre avec un coefficient de frottement $\rho=0,28$. Cette étude a été réalisée sur un lot de plaquettes de PMMA ayant différentes inclinaisons de défaut $\beta$ variables allant de 15 à $75^{\circ}$ par rapport à la direction de la charge.

En mesurant la distance $\delta$ du front de l'entaille à la racine du branchement pour des défauts d'inclinaison $\beta$ allant de 30 à $75^{\circ}$, on a remarqué que la distance $\delta$ augmente d'une part avec l'inclinaison $\beta$ du défaut de 0,32 à $0,5 \mathrm{~mm}$ (Fig. 17), et d'autre part avec le coefficient de frottement $\rho$.

Pour la mesure de l'angle de branchement $\theta$ entre la direction de l'entaille et la tangente à la racine de la fissure branchée, on a constaté que l'angle de branchement $\theta$ croît avec l'angle d'inclinaison du défaut $\beta=30$ et $45^{\circ}$ à partir de la valeur moyenne $\theta_{\mathrm{m}}=69^{\circ}, 37$ pour $\beta=30^{\circ}$ jusqu'à $\theta_{\mathrm{m}}=76^{\circ}, 42$ pour $\beta=45^{\circ}$ (Fig. 18). Par contre, l'angle de branchement $\theta$ augmente et tend vers la valeur critique $\theta_{\mathrm{c}}=80 \pm 1^{\circ}$ par rapport à la direction de l'entaille pour les inclinaisons $\beta$ allant de 60 à $75^{\circ}$ (Fig. 18). Aussi, on a remarqué que l'angle de branchement $\theta$ diminue en augmentant le coefficient de frottement $\rho$ et qu'il n'est plus perpendiculaire au plan de la fissure, contrairement au cas d'un défaut ouvert (pas de frottement) où la fissure branchée s'initie avec un angle de branchement $\theta$ qui tendait rapidement à $90^{\circ}$.

\section{Conclusions}

À partir de cette étude expérimentale, dégageons les points les plus importants. D'abord, dès qu'on applique une pression suffisante sur des plaquettes de PMMA fissurées présentant un défaut oblique et fermé en présence $\mathrm{du}$ frottement pour des différentes inclinaisons $\beta \mathrm{du}$ 


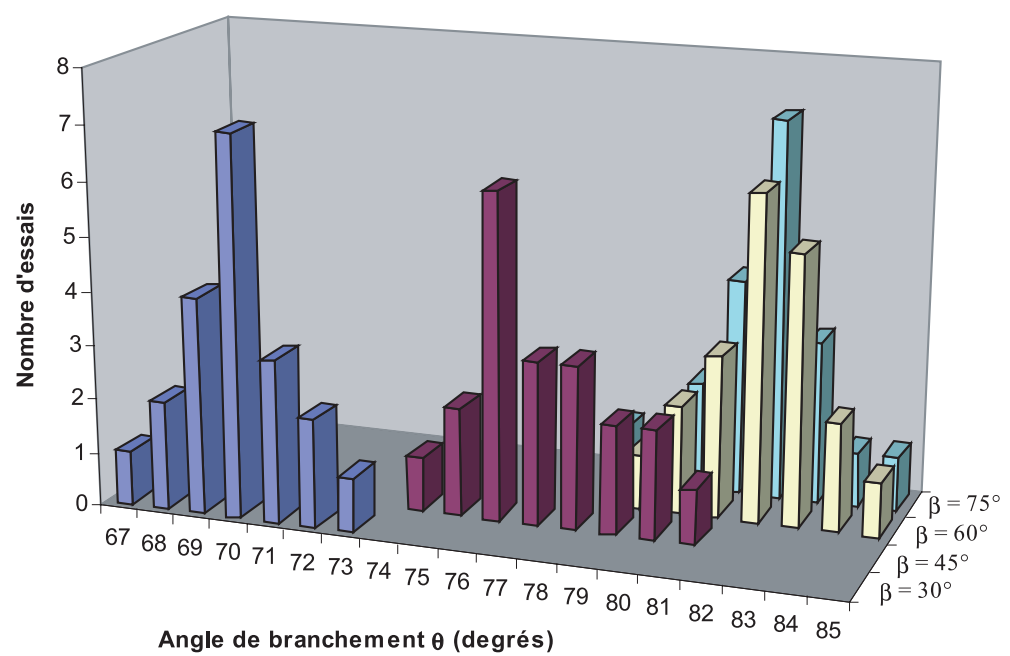

Fig. 16. Histogrammes représentant l'angle de branchement $\theta$ pour les inclinaisons $\beta$ variables de 30 à $75^{\circ}$ en présence d'aluminium $(\rho=0,18)$.
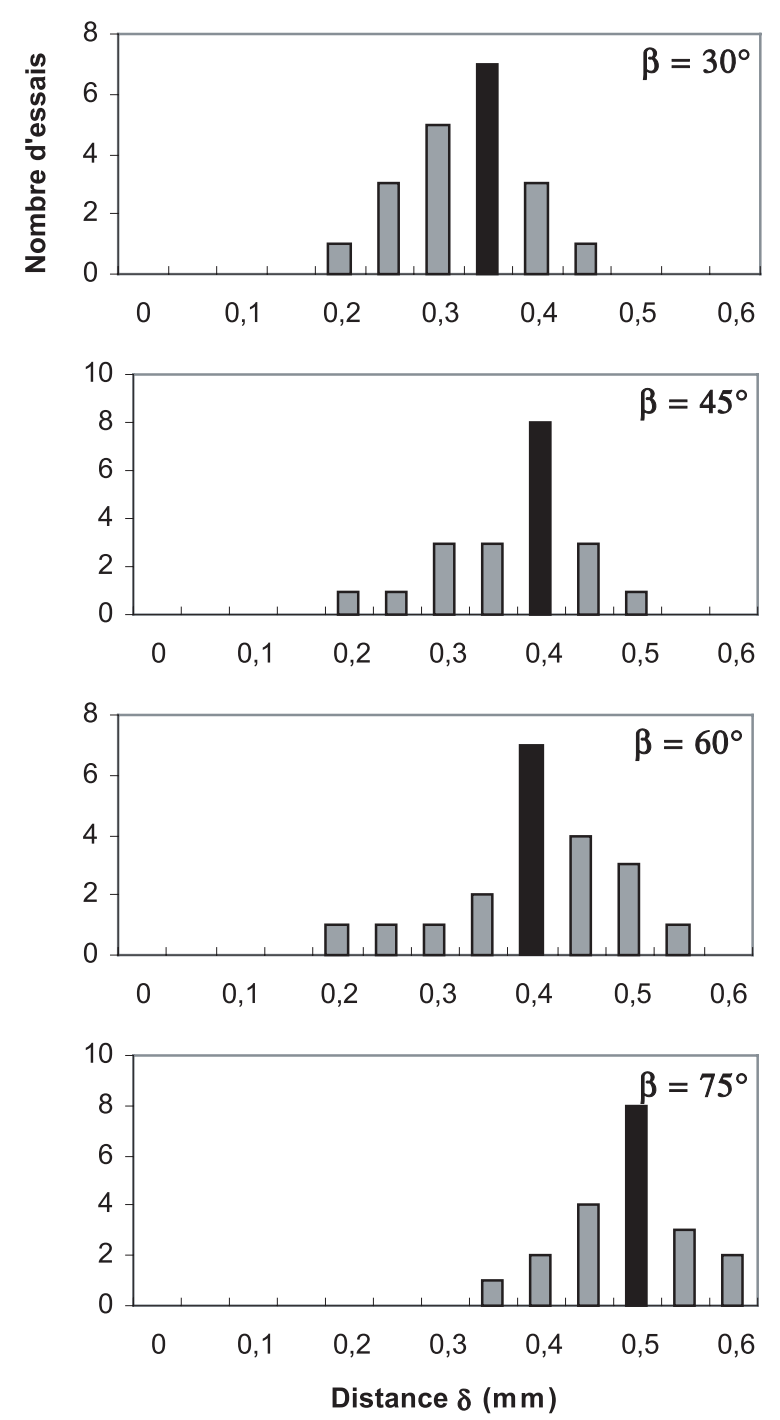

Fig. 17. Histogrammes représentant la distance $\delta$ pour les inclinaisons $\beta$ allant de 30 à $75^{\circ}$ en présence de cuivre $(\rho=$ $0,28)$. défaut variables de 15 à $75^{\circ}$ par rapport à la direction de la compression, on observe la propagation de deux fissures branchées, symétriques par rapport au centre du défaut et qui rejoignent la direction de la charge imposée. Ensuite, on a constaté que la fissure branchée est toujours initiée à une distance $\delta$ non nulle de l'extrémité du défaut fermé et croît avec l'inclinaison $\beta$ du défaut par rapport à la direction du chargement uniaxial, ce comportement est identique à celui du défaut ouvert, pour lequel le frottement est nécessairement nul.

Pour un coefficient de frottement donné, la fissure branchée s'ouvre d'autant plus loin de l'extrémité du défaut fermé que l'inclinaison est plus grande. Compte tenu du mode de façonnage des entailles par sciage, il se produit un rayon de courbure $R$ qui est très petit dans le plan médian de symétrie de la surface de l'éprouvette. Ainsi, on comprend la géométrie de la fissure branchée au voisinage du front du défaut et la concentration des valeurs expérimentales des distances. Cependant, en présence de micro-fractures, de profondeur importante sur le bord du défaut, la distance $\delta$ de démarrage est celle qui correspond au lieu de micro-fracture où le taux de restitution d'énergie $G$ est maximum, ce qui explique les quelques mesures expérimentales des distances $\delta$ assez éloignées des valeurs moyennes.

Il en est de même pour la valeur moyenne de l'angle de branchement $\theta$ qui augmente avec l'angle d'inclinaison $\beta$. Pour les deux cas : défaut ouvert et défaut fermé avec frottement, les dispersions des mesures des deux paramètres $\delta$ et $\beta$ étant de même amplitude et ce qui diffère, du fait de l'existence d'un cisaillement au niveau des lèvres fermées du défaut initial, conduisant à un coefficient de frottement à l'aide d'une mesure parallèle, est la valeur limite $\theta_{c}$ atteinte par l'angle de branchement $\theta$ qui tendait rapidement vers $90^{\circ}$ en absence de frottement, tend ici asymptotiquement vers la valeur critique $\theta_{\mathrm{c}}$ qui dépend de la nature du matériau, d'où l'influence du frottement sur l'initiation de la fissure branchée déterminée par l'angle de branchement $\theta$. 


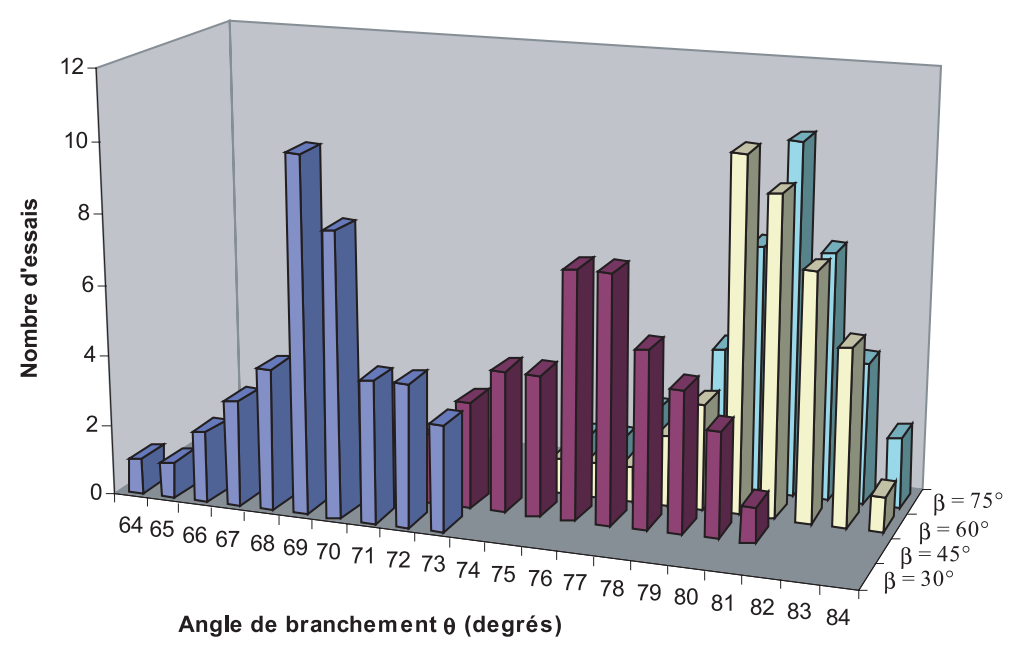

Fig. 18. Histogrammes représentant l'angle de branchement $\theta$ pour les inclinaisons $\beta$ variables de 30 à $75^{\circ}$ en présence de cuivre $(\rho=0,28)$.

De plus, on a bien mis en évidence que la distance $\delta$ du front de l'entaille à la racine du branchement croît avec le coefficient de frottement $\rho$, par contre l'angle de branchement $\theta$ entre la direction de l'entaille et la tangente à la racine de la fissure branchée diminue au fur et à mesure que le coefficient de frottement $\rho$ augmente.

\section{Références}

[1] J.-P. Petit, M. Barquins, Formation de fissures en milieu confiné dans le PMMA (polyméthacrylate de méthyle) : modèle analogique de formation de structures tectoniques cassantes, Comptes Rendus de l'Académie des Sciences de Paris, t. 305, série II, 1987, pp. 55-60

[2] J.-P. Petit, M. Barquins, Can natural faults propagate under mode II conditions?, Tectonics 7 (1988) 1243-1256

[3] P.B. Bowden, S. Raha, The formation of micro-shear bands in polystyrene and polymethylmethacrylate, Phil. Mag. 22 (1970) 463-482

[4] M. Barquins, J.-P. Petit, D. Maugis, K. Ghalayini, Path and kinetics of branching from defects under uniaxial and biaxial compressive loading, Int. J. Fracture 54 (1992) 139-163
[5] J.-P. Petit, M. Barquins, Localisation des bandes de cisaillement par un défaut isolé préexistant : données expérimentales, Bull. Soc. Géol. France 164(2) (1993) 255-266

[6] C. Chaker, M. Barquins, Sliding effect on branch crack, Physics and Chemistry of the Earth 21(4) (1996) 319-323

[7] M. Barquins, C. Chaker, J.-P. Petit, Influence du frottement sur le branchement de fissures à partir de défauts obliques soumis à une compression uniaxiale, Comptes Rendus de l'Académie des Sciences de Paris, t. 324, Série IIb, 1997, pp. 29-36

[8] C. Chaker, Étude du champ des contraintes en présence $\mathrm{du}$ frottement, Revue Française de Mécanique $\mathrm{n}^{\circ}$ 2001-1 (2001) 9-14

[9] C. Chaker, Stress trajectories in the presence of friction, Int. J. Sol. Struc. 40(15) (2003) 4033-4041

[10] J.-P. Petit, M. Barquins, Fault propagation in Mode II conditions: Comparison between experimental and mathematical models, applications to natural features, Mechanics of Jointed and Faulted Rock, A.A. Balkema, Brookfield, Rotterdam, 1990, pp. 213-220

[11] M. Barquins, J.-P. Petit, Path and propagation kinetics of branch fractures formed in brittle materials, in mode II conditions, Mechanical Properties, Materials Design, 1991, pp. 3-11 